\title{
AVALIAÇÃO DOS MARCOS DO DESENVOLVIMENTO INFANTIL SEGUNDO A ESTRATÉGIA DA ATENÇÃO INTEGRADA ÀS DOENÇAS PREVALENTES NA INFÂNCIA
}

\author{
Evaluation of the mark of child development according to strategy integrated \\ management of childhood illness \\ Evaluación de los marcos de desarrollo infantil según la estrategia de atención \\ integrada a las enfermedades prevalentes de la infancia
}

\begin{abstract}
RESUMO
A pesquisa objetiva avaliar o desenvolvimento de crianças de 0 a 24 meses matriculadas em uma creche de Ipatinga, Minas Gerais, através dos marcos do desenvolvimento infantil propostos pela Atenção Integrada às Doenças Prevalentes na Infância. Trata-se de um estudo descritivo com análise quantitativa dos dados. A amostra foi composta por 20 crianças de 0 a 24 meses, e os dados foram coletados no período de 13 de fevereiro a 6 de março de 2009. Das 20 crianças avaliadas, 4 (20\%) foram classificadas com "Possível atraso"; 1 (5\%), com "Provável atraso"; e 15 (75\%), com "Desenvolvimento normal". Os resultados encontrados demonstraram que a ficha de acompanhamento do desenvolvimento proposta pelo Manual para Vigilância do Desenvolvimento Infantil constitui-se em um instrumento de triagem de fácil aplicação, de baixo custo operacional e capaz de realizar a detecção precoce dos atrasos, devendo, portanto, fazer parte das ações do enfermeiro na consulta de puericultura.
\end{abstract}

Palavras-chave: Desenvolvimento Infantil. Creches. Atenção Integrada às Doenças Prevalentes na Infância.

\begin{abstract}
The research objective to evaluate the development of children 0 to 24 months enrolled in a kindergarten of Ipatinga, Minas Gerais, through the milestones of child development proposed by Integrated Management of Childhood Illness. This is a descriptive study with quantitative data analysis. The sample comprised 20 children from 0 to 24 months and data were collected from February 13 to March 6, 2009. Of the 20 children evaluated, 4 (20\%) were classified as "possible delay," 1 (5\%) as "Probable delay and 15 (75\%) as" normal development ". The results showed that the form of monitoring of the development proposed by the Manual for Monitoring of Child Development is itself a tool for easy screening of application, low cost operational and able to perform early detection of delays and should therefore do part of the actions of the nurse in consultation with childcare.
\end{abstract}

Keywords: Child development. Child Day Care Centers. Integrated Management of Childhood IIIness.

\section{Resumen}

La investigación tiene por objetivo evaluar el desarrollo de niños de 0 a 2 meses matriculados en una guardería en Ipatinga, Minas Gerais, a través de los hechos importantes del desarrollo infantil propuesto por la Atención Integrada a las Enfermedades Prevalentes de la Infancia. Este es un estudio descriptivo con el análisis de datos cuantitativos. La muestra comprende 20 niños de 0 a 24 meses y los datos fueron recolectados desde el 13 de febrero hasta el 06 de marzo del año de 2009. De los 20 niños evaluados, 4 (20\%) fueron clasificados como "posible retraso", 1 (5\%) como "probable retraso" y 15 (75\%) como "desarrollo normal". Los resultados mostraron que la ficha de acompañamiento propuesta por el Manual para la Vigilancia del Desarrollo Infantil se constituye en una herramienta de triar de fácil aplicación, bajo costo operacional y capaz de llevar a cabo la detección precoz de retrasos y, por lo tanto, hacer parte de las acciones de la enfermera en la consulta de puericultura.

Palabras clave: Desarrollo infantil. Jardines Infantiles. Atención Integrada a las Enfermedades Prevalentes de la Infancia.

\footnotetext{
${ }^{1}$ Mestre em Enfermagem pela Faculdade de Enfermagem da UERJ. Especialista em Enfermagem em Saúde Pública pela Escola de Enfermagem da UFMG. Enfermeira do CEREST/Ipatinga. Docente do Centro Universitário do Leste de Minas Gerais - Ipatinga.-MG- Brasil. E-mail: marciaelenaenf@yahoo.com.br,²Enfermeira PósGraduanda em Enfermagem do Trabalho pelo Centro Universitário do Leste de Minas Gerais Unileste -Ipatinga- MG. Brasil. E-mail: nayaratq@hotmail.com, ${ }^{3}$ Enfermeira Especialista em Saúde Pública Interdisciplinar pela UNIFOA-Rio de Janeiro-RJ. Brasil. E-mail: renataxalmeida@yahoo.com.br
} 


\section{INTRODUÇÃO}

A sobrevivência infantil é uma das principais questões que ficaram pendentes no século XX. Esse fato resulta em uma preocupação com o crescimento e desenvolvimento infantil no novo século, no intuito de contribuir para o decréscimo dos índices de morbimortalidade infantil e repercutir positivamente na melhoria das condições gerais de vida da população. ${ }^{1}$

A infância é o período da vida no qual a preocupação com o desenvolvimento deve ser prioritária. Tal fato se dá porque, nesta etapa da vida, os indivíduos estão com o sistema nervoso em intenso desenvolvimento, sendo, portanto, mais susceptíveis às doenças ou a agravos à saúde. ${ }^{2}$

A preocupação com o desenvolvimento infantil passou a fazer parte das ações de promoção em saúde da criança propostas pela Organização Pan-Americana de Saúde (OPAS) em meados da década de 90 , a partir da criação da estratégia de Atenção Integrada às Doenças Prevalentes na Infância (AIDPI). ${ }^{1}$

Tal estratégia foi criada com o objetivo de capacitar os profissionais de saúde para prestar uma assistência integral à saúde das crianças e fornecer às famílias conhecimentos científicos básicos que auxiliem na promoção da saúde e prevenção de agravos nos primeiros anos de vida. ${ }^{1}$

A AIDPI foi implantada no Brasil e constitui-se, atualmente, uma prioridade das políticas de saúde do Ministério da Saúde (MS) para a população infantil, com ênfase na atenção primária. Tem como objetivo reduzir a mortalidade na infância, por meio da melhoria na qualidade do atendimento prestado às crianças, baseando-se na capacitação dos profissionais, organização dos serviços e educação da família. ${ }^{1}$

A implementação da AIDPI no Brasil está sendo realizada no contexto da Estratégia Saúde da Família (ESF). Iniciou-se em 1997 pela região nordeste e tem avançado rapidamente pelo território nacional. 0 motivo da escolha do nordeste como área prioritária deve-se ao fato de essa região sempre ter apresentado os piores indicadores socioeconômicos e de saúde entre a população infantil brasileira, o que justifica a realização de um rigoroso acompanhamento do crescimento e desenvolvimento das crianças que ali vivem. ${ }^{1}$

A partir daí, a OPAS, juntamente com a Organização Mundial de Saúde (OMS), desenvolveu o Manual de Vigilância do Desenvolvimento Infantil no contexto da AIDPI. Inicialmente, ele surgiu para suprir a necessidade de capacitação dos profissionais que atuavam na Secretaria Municipal de Saúde de Belém-PA, em relação ao desenvolvimento infantil. Esta experiência apresentou resultados positivos e, atualmente, está sendo incorporada na atenção à saúde da criança em vários estados no Brasil e também em outros países. ${ }^{1}$

0 Desenvolvimento Infantil (DI) são mudanças nas estruturas físicas, neurológicas, cognitivas e comportamentais do indivíduo que ocorrem de forma ordenada e relativamente duradouras, ${ }^{3}$ pode ser entendido, também, como mudanças nas funções corporais, incluindo aquelas influenciadas por fatores emocionais e sociais. ${ }^{4}$ Para OMS este é um processo que ocorre desde a concepção do feto até a morte, envolve o crescimento físico, a maturação neurológica, comportamental, cognitiva, social e afetiva da criança, tendo como produto um indivíduo "competente para responder às suas necessidades e às do seu meio, considerando seu contexto de vida"::12.

Nesta perspectiva, é de extrema importância considerar os fatores de risco para o desenvolvimento na avaliação de saúde da criança para que se possam estabelecer prioridades nas ações voltadas para a diminuição da incidência desses agravos entre a população infantil.?

Os desvios no desenvolvimento, quando comparados aos padrões de normalidade estabelecidos, trazem indícios de que algo está errado, mesmo quando os pais ou responsáveis ainda não perceberam o problema. Deve-se fazer uma avaliação do peso, da estatura e do perímetro cefálico. Caso haja discrepância alta entre os seus percentis, o profissional deve ficar atento para algum desvio. ${ }^{4}$

A AIDPI preconiza uma série de marcos do desenvolvimento que devem ser cumpridos pelas crianças durante uma avaliação. Caso a criança não consiga realizar alguma atividade proposta, a mãe será orientada e/ou a criança deverá ser referenciada para uma unidade de saúde de maior complexidade, dependendo da gravidade detectada na consulta. Como instrumento de avaliação do desenvolvimento infantil, a estratégia AIDPI colocou à disposição das famílias um conjunto básico de intervenções que permitem aos profissionais de saúde promover a prevenção, a detecção precoce das alterações e 0 tratamento dos principais agravos à saúde durante a infância, além de aplicar medidas de promoção da saúde integral ao proporcionar conhecimento e prática para os pais, na atenção ao cuidado de seus filhos. ${ }^{1}$

Este instrumento utilizado pela AIDPI compreende uma estratégia de triagem que fornece resultados eficazes para determinar se a criança está se desenvolvendo de acordo com o esperado para sua idade cronológica e maturidade, além de detectar atrasos no desenvolvimento através de alguns reflexos, posturas, habilidades, desenvolvimento motor fino e grosso, linguagem e interação pessoal. A ausência no cumprimento de um só marco já é considerada desvio para tomada de providências. ${ }^{1}$

A vigilância do desenvolvimento infantil deve ser realizada na atenção primária, através das atividades de promoção do desenvolvimento normal e da deteç̧ão precoce de problemas ou alterações. Diante disso, torna-se importante afirmar que os profissionais de saúde devem possuir conhecimentos básicos sobre o desenvolvimento infantil, para que possam fazer o seu acompanhamento de forma sistemática para todas as crianças sob sua responsabilidade, a fim de decidir se há um problema para ser investigado e qual tratamento adequado. 
Não existem dados estatísticos confiáveis que apontem a real incidência de crianças com problemas no desenvolvimento, uma vez que a classificação de uma criança com desenvolvimento normal é considerada complexa. ${ }^{1} \mathrm{De}$ acordo com estimativas da OMS, $10 \%$ da população de qualquer país são constituídos por pessoas com algum tipo de deficiência. ${ }^{5}$ Considerando a população brasileira do censo de 2007 , de 183.987.291 habitantes, teríamos, portanto, 18.398.729 pessoas deficientes, estando, entre elas, crianças com atraso no desenvolvimento. ${ }^{5}$

Diante da magnitude que esses dados representam para a população infantil brasileira, justificou-se a realização dessa pesquisa com o intuito de conscientizar os profissionais de saúde sobre a importância da vigilância do desenvolvimento infantil através da estratégia AIDPI, a fim de detectar precocemente a ocorrência de variações para oferecer orientações à família e, se necessário, fazer o encaminhamento para diagnóstico e intervenção.

Um desenvolvimento infantil adequado contribui para a geração de indivíduos com maior senso crítico, aptos a enfrentarem dificuldades, tornarem-se adolescentes, jovens e adultos sadios e socialmente produtivos. ${ }^{1}$ Já existem, atualmente, pesquisas suficientes capazes de afirmar que, quanto mais cedo o diagnóstico de atraso no desenvolvimento infantil é realizado, menores serão os danos causados a essa criança. ${ }^{6}$

Neste contexto, a AIDPI tem como tarefa essencial a implementação de intervenções adequadas, que contribuam para a prevenção de doenças, para sua deteç̧ão precoce e tratamento eficaz, bem como para a promoção da saúde integral da população infantil. Ao implantá-la no município, os gestores estarão assumindo um compromisso ético em trabalhar pela sobrevivência infantil e a favor da infância saudável. ${ }^{1}$

Baseado nessas afirmativas, este estudo teve como objetivo avaliar o desenvolvimento de crianças de 0 a 24 meses, matriculadas em uma creche do município de Ipatinga-MG, através dos marcos do desenvolvimento infantil propostos pela AIDPI.

\section{METOLOGIA}

Trata-se de um estudo descritivo com análise quantitativa dos dados, realizado na Creche Lar Escola da Caridade, localizada na Rua Nova Almeida, $n^{0} 158$, no bairro Vila Ipanema, em Ipatinga, Minas Gerais. É uma instituição comunitária, conveniada com a Secretaria Municipal de Educação, tendo como público-alvo uma população de 102 crianças com idades entre de 0 a 5 anos incompletos, de famílias carentes, cujas mães são trabalhadoras, ou a família é de risco social.

A instituição foi selecionada como campo de investigação porque há 4 anos é realizado nesta o projeto de extensão "Creche Saudável" do curso de Enfermagem do Centro Universitário do Leste de Minas Gerais (Unileste-MG) sob a coordenação da pesquisadora principal deste estudo, além de abrigar crianças em situações diversas e singulares, importantes para o que se propôs esta pesquisa.

A amostra estudada foi composta por 20 crianças de 0 a 24 meses que estiveram presentes no momento da avaliação e foram autorizadas pelo responsável a participarem do estudo. A idade das crianças foi selecionada como critério de inclusão na amostra por pertencer a faixa etária proposta para a avaliação do desenvolvimento de acordo com o preconizado pela estratégia AIDPI. Foram excluídas da amostra 82 crianças com idade superior a 24 meses.

0 instrumento de coleta de dados foi a ficha de acompanhamento do desenvolvimento proposta pelo Manual para Vigilância do Desenvolvimento Infantil no Contexto da AIDPI. ${ }^{1}$ Esta ficha propõe que a avaliação do desenvolvimento infantil deve seguir as seguintes etapas: (1) levantar informações com as mães ou responsáveis sobre fatores de risco para o atraso no desenvolvimento, (2) identificar a percepção da mãe sobre o desenvolvimento do seu filho, (3) verificar as medidas do perímetro cefálico e (4) verificar a presença de alterações fenotípicas através do exame físico. Além disso, deve-se avaliar a postura, os comportamentos e reflexos, de acordo com a faixa etária da criança.

As crianças da amostra na faixa etária de 2 meses a 2 anos de idade devem ser avaliadas em 32 marcos do desenvolvimento infantil, divididos por oito faixas etárias compreendendo períodos de 2 a 24 meses de vida $(2,4,6,9$, $12,15,18$ e 24 meses) e considerando-se que esses marcos devem estar presentes em $90 \%$ das crianças para esta faixa etária (percentil 90). ${ }^{1}$

Nesta avaliação foram consideradas quatro áreas para observação: motora grossa, motora fina, linguagem e interação pessoal-social. A ausência no cumprimento de um só marco foi considerada desvio significativo para tomada de decisão, de acordo com a faixa etária da criança. ${ }^{1}$

A avaliação do desenvolvimento foi realizada no consultório de enfermagem, no horário de 13 às 17 horas, com duração aproximada de 30 minutos para cada uma. As crianças que se apresentaram resistentes e agitadas nesse momento foram levadas à sua sala e avaliadas próximas à professora responsável no intuito de minimizar as intercorrências e facilitar a interpretação das respostas apresentadas.

Após a coleta, os dados foram categorizados, codificados, registrados em uma planilha do Excel e 
avaliados através da comparação com a proposta da AIDPI. Em seguida, eles foram submetidos a tratamento estatístico, dispostos em tabelas e gráficos e comparados com a literatura pesquisada para a construção dos resultados e discussões.

0 estudo foi submetido aos procedimentos normativos do Comitê de ética ${ }^{7}$ em pesquisa do Centro Universitário do Leste de Minas Gerais, aprovado sob o protocolo número 22.128.08-CEP/ Unileste-MG.

\section{RESULTADOS E DISCUSSÃO}

Fizeram parte deste estudo 20 crianças com idade entre 0 e 2 anos incompletos, sendo 9 (45,0\%) do sexo feminino e $11(55,0 \%)$ do sexo masculino. A Tabela 1 demonstra a distribuição das crianças segundo exposição aos fatores de risco para o atraso no desenvolvimento. Das 20 mães ou responsáveis entrevistadas, 19 (95,0\%) informaram que realizaram as consultas de pré-natal e $1(5,0 \%)$ não soube informar em função de seu filho ser adotivo.

Tabela 1-Distribuição das crianças segundo a exposição aos fatores de risco para o atraso no desenvolvimento. Ipatinga, MG, 2009.

\begin{tabular}{|c|c|c|c|c|c|c|c|c|}
\hline \multirow{2}{*}{ FATORES DE RISCO } & \multicolumn{2}{|c|}{ SIM } & \multicolumn{2}{|c|}{ NÃO } & \multicolumn{2}{|c|}{ NÃO SABE } & \multicolumn{2}{|c|}{ TOTAL } \\
\hline & $\mathbf{N}$ & $\%$ & $\mathbf{N}$ & $\%$ & $\mathbf{N}$ & $\%$ & $\mathbf{N}$ & $\%$ \\
\hline Realizou o pré-natal? & 19 & 95,0 & 0 & 0 & 1 & 5,0 & 20 & 100 \\
\hline Problema durante a gestação, parto ou nascimento? & 3 & 15,0 & 16 & 80,0 & 1 & 5,0 & 20 & 100 \\
\hline Prematuridade? & 3 & 15,0 & 17 & 85,0 & 0 & 0 & 20 & 100 \\
\hline $\begin{array}{l}\text { Patologias do filho: meningite, traumatismo craniano ou } \\
\text { convulsão? }\end{array}$ & 0 & 0 & 20 & 100 & 0 & 0 & 20 & 100 \\
\hline Consanguinidade? & 2 & 10,0 & 18 & 90,0 & 0 & 0 & 20 & 100 \\
\hline Histórico familiar de deficiência mental? & 2 & 10,0 & 18 & 90,0 & 0 & 0 & 20 & 100 \\
\hline $\begin{array}{l}\text { Violência doméstica, depressão materna, drogas e } \\
\text { alcoolismo? }\end{array}$ & 1 & 5,0 & 19 & 95,0 & 0 & 0 & 20 & 100 \\
\hline Presença de alterações fenotípicas? & 0 & 0 & 20 & 100 & 0 & 0 & 20 & 100 \\
\hline
\end{tabular}

A cobertura de pré-natal no município de Ipatinga no ano de 2006 apresentou a seguinte situação: $47,8 \%$ das mulheres realizaram sete consultas ou mais, $40 \%$ tiveram 4 a 6 consultas e $10 \%$ realizaram menos de 4 consultas no período gestacional. ${ }^{8}$ Esta realidade reflete os bons índices de cobertura do atendimento das mulheres grávidas do município, estando de acordo com o proposto pelo Ministério da Saúde.

0 Ministério da Saúde preconiza a realização de 6 consultas durante o pré- natal, sendo: 1 consulta no primeiro trimestre; 2 consultas no segundo trimestre; e 3 consultas no terceiro trimestre. Uma atenção ao pré-natal, com qualidade e humanização, é necessária para que haja uma mudança no processo saúde-doença. Essa mudança depende de novos olhares dos profissionais, gestores e usuários, levando-se em consideração o ambiente cultural, social e econômico nos quais as pessoas vivem. Além disso, sabe-se que o aumento do número de visitas pré-natais, aliado a cuidados precoces oferecidos às gestantes, pode reduzir as taxas de nascimento prematuro e de fetos de baixo peso. Vale acrescentar que a frequência das visitas e o momento em que a primeira delas é realizada devem ser avaliados, pois interferem nas taxas de mortalidade perinatal. ${ }^{8}$

Apenas $3(15,0 \%)$ mães relataram problemas durante a gestação (pré-eclâmpsia, dispneia, dor lombar, edema nas pernas), parto ou nascimento, 1 (5,0\%) não soube informar e $16(80,0 \%)$ negaram qualquer problema neste período (Tabela 1). A maior parte dos óbitos em crianças se concentra no primeiro mês de vida, demonstrando a importância de se considerar fatores ligados à gestação, parto ou nascimento.

Enquanto os índices de doenças infecciosas, parasitárias e respiratórias e de desnutrição infantil diminuíram no Brasil, nas últimas décadas, as afecções perinatais, originadas até a primeira semana de vida (como prematuridade, asfixia e infecções neonatais) tornaram-se cada vez mais frequentes, chegando, em 2003, a representar 55\% das causas de mortalidade infantil. Para reverter essa situação é necessário investir em um acompanhamento pré-natal adequado, além de reforçar os cuidados com o parto e nascimento da criança. ${ }^{9,10}$

Dentre as crianças da amostra, 17 (85,0\%) nasceram a termo e 3 (15,0\%) nasceram pré-termo. 0 recém-nascido 
(RN) pré-termo está mais susceptível a problemas logo após o nascimento ou mais tardiamente, tais como: hipóxia, síndrome da membrana hialina, doença hemorrágica, hemorragias intracranianas, infecções, hipoglicemia, hipovitaminoses (A, D, $\mathrm{E}, \mathrm{K})$ e atraso do desenvolvimento neuropsicomotor. ${ }^{2}$

A prematuridade é decorrente de diversos fatores e causas e está presente em todas as classes sociais. Geralmente acarreta às famílias um alto custo social e financeiro, além de exigir das instituições e dos profissionais de saúde uma grande estrutura assistencial e técnica, nem sempre disponíveis nos serviços de saúde de nosso país. A fragilidade dos recém-nascidos prematuros contribui para o surgimento de complicações que exigem diferentes intervenções na estimulação do processo de crescimento e desenvolvimento infantil, tornando-se necessário prever riscos para que se possam instaurar medidas preventivas. ${ }^{11}$

Com relação aos agravos como convulsões, meningite e traumatismo craniano, não houve nenhum relato de caso entre as crianças estudadas (Tabela 1). Porém, é importante abordar essas patologias devido ao risco de sequelas, como atrasos no desenvolvimento neuropsicomotor.

0 ataque convulsivo reflete uma patologia que pode influenciar diretamente no desenvolvimento infantil, por ser uma indicação de comprometimento da integridade do sistema nervoso central. ${ }^{12}$

A meningite é uma doença muito grave. Apesar de a terapia antimicrobiana ter avançado, ainda existem inúmeros casos em crianças. Essa doença ocorre mais comumente no período de lactação e na segunda infância, contribuindo para um aumento na taxa de mortalidade. 0 seu prognóstico depende da resposta do indivíduo e do fator causador da doença. De uma maneira geral poderá haver comprometimento visual, surdez, convulsões, paralisia e choque séptico, comprometendo, portanto, o desenvolvimento infantil. ${ }^{4}$

0 traumatismo craniano constitui um dos maiores problemas de saúde pública, principalmente nos países desenvolvidos. Antes dos 15 anos, 50\% dos óbitos nos Estados Unidos ocorrem devido a traumatismo craniano. Entre as crianças, quando não evoluem para o óbito, uma parcela significativa apresenta dificuldades comportamentais e de aprendizagem..$^{13} 0$ traumatismo craniano em crianças pode, ainda, levar à depressão da consciência, com ou sem déficit neurológico. Essa depressão demanda tratamento neurocirúrgico e o déficit neurológico exige observação neurológica. ${ }^{4}$

Quanto ao grau de parentesco dos pais, 2 $(10,0 \%)$ mães afirmaram ser parentes dos pais das crianças e $18(90,0 \%)$ negaram tal parentesco. A consanguinidade entre os pais é um aspecto que favorece o aparecimento de doenças autossômicas recessivas que podem levar ao atraso do desenvolvimento infantil. Sendo assim, a coleta do histórico familiar é necessária, principalmente em crianças que nascem com malformação, para auxiliar e esclarecer se os problemas apresentados são de origem genética. ${ }^{2}$

Nesta pesquisa, os resultados mostraram que existem $2(10,0 \%)$ casos de deficiência mental na família das crianças, e $18(90,0 \%)$ negaram histórico familiar desse agravo. A deficiência mental é uma condição patológica do cérebro que leva ao processo de doença, podendo surgir em qualquer época do período do desenvolvimento. ${ }^{12} 0$ desenvolvimento psíquico do ser humano se constitui a partir da vivência de suas relações e trocas com o outro, ocorre ao longo da vida, desde a mais tenra infância. Portanto, a influência das pessoas que convivem com a criança, principalmente no meio familiar, merece destaque, tornando-se imprescindível o questionamento acerca de casos de deficiência mental e/ ou física na família. ${ }^{6}$

Em relação ao uso de drogas e álcool, 1 (5\%) mãe relatou que seu filho vivencia essa situação com o pai, que chega em casa sempre alcoolizado e, às vezes, violento. A violência contra a criança caracteriza-se por qualquer forma de agressão ou omissão que provoque danos, lesões ou transtornos a seu desenvolvimento. ${ }^{9}$

As manifestações físicas do abuso infantil se apresentam como atraso do crescimento, distúrbios alimentares e distúrbios do sono. Além disso, a criança pode apresentar comportamentos sugestivos, como os autoestimulatórios: morder; balançar e sugar. Durante a lactância, a ausência de sorriso social e ansiedade na presença de estranhos também podem gerar suspeita de violência. A criança se isola, sente um medo incomum, mantém um compor tamento antissocial (com características destrutivas, de furto e crueldade), manifesta extremos de comportamento (excessivamente obediente e passiva ou agressiva e exigente), podendo, ainda, apresentar retardo no desenvolvimento emocional e intelectual, principalmente linguístico. ${ }^{14}$

As $20(100 \%)$ crianças avaliadas não apresentaram alterações fenotípicas. 0 fenótipo de uma pessoa se manifesta através de suas características morfológicas, resulta da expressão dos genes do organismo, da influência dos fatores ambientais e pela possível interação entre os dois. As pessoas que apresentam cromossomos com anormalidades têm fenótipos característicos. Crianças com síndrome de Down, por exemplo, assemelham-se mais a outras pessoas que apresentam a mesma anormalidade do que com seus próprios pais e irmãos. ${ }^{15}$

0 Gráfico 1 apresenta a percepção da mãe sobre 0 desenvolvimento do seu filho. 10 (50,0\%) mães classificaram como bom o desenvolvimento infantil do seu filho, $2(10,0 \%)$ classificaram como ótimo e 8 (40,0\%), como normal. A mãe geralmente é a pessoa que mais convive com a criança, sendo, portanto, quem mais a observa. Assim, é capaz de saber se seu filho está se desenvolvendo como as crianças à sua volta 
ou apresenta alguma dificuldade. A opinião da mãe auxilia muito na avaliação da criança, pois se ela disser que algo não vai bem, o cuidado com seu filho deve ser redobrado. ${ }^{1}$

Gráfico1 - Classificação do desenvolvimento das crianças segundo avaliação das mães. Ipatinga, MG, 2009.

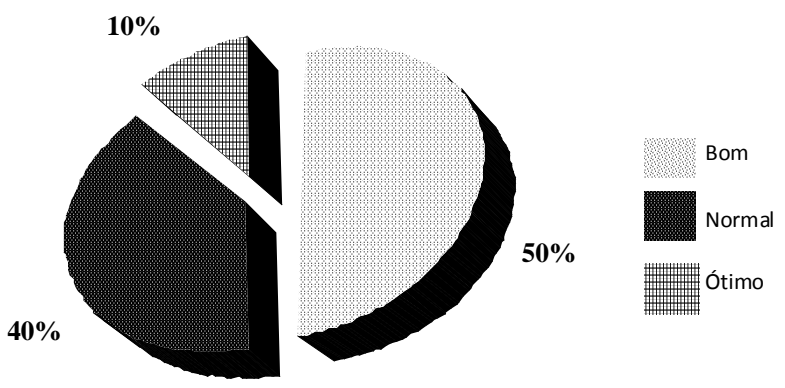

Verifica-se na Tabela 2 que o peso das crianças ao nascer variou de 600 a 4.225 gramas, sendo que $3(15,0 \%)$ delas foram classificadas como de baixo peso (inferior a 2.500 gramas).

Tabela 2 - Distribuição das crianças estudadas em relação ao peso de nascimento. Ipatinga, MG, 2009.

\begin{tabular}{lcc}
\hline PESO AO NASCER (Kg) & N & $\%$ \\
\hline$=3,5$ & 4 & 20,0 \\
$3,00-3,49$ & 9 & 45,0 \\
$2,50-2,99$ & 3 & 15,0 \\
$2,00-2,49$ & 0 & 0 \\
$<2,00$ & 3 & 15,0 \\
Não sabe informar & 1 & 5,0 \\
\hline TOTAL & 20 & 100 \\
\hline
\end{tabular}

0 peso ao nascer reflete as condições nutricionais do recém-nascido e da gestante e é considerado um indicador de saúde individual. 0 baixo peso ao nascer, caracterizado pela OMS como abaixo de 2.500 gramas, é um fator de risco para o aumento da morbimortalidade infantil e está, geralmente, associado à prematuridade ou, ainda, ao retardo do crescimento intra uterino. ${ }^{16}$

Observa-se na Tabela 3 o valor do perímetro cefálico das crianças da amostra de acordo com o percentil por faixa etária. Esses valores estão de acordo com o Manual do Acompanhamento do Crescimento e Desenvolvimento Infantil proposto pelo Ministério da Saúde; 1 (5,0\%) apresentou o percentil menor que 10, valor abaixo do esperado para idade (considerado fora da faixa de normalidade); 19 (95,0\%), o percentil entre 10 e 90 , adequado para idade (faixa de normalidade); e nenhuma criança apresentou percentil maior que 90 , acima do esperado para idade (fora da faixa de normalidade).
Tabela 3 - Distribuição das crianças estudadas em relação ao percentil do perímetro cefálico no momento do exame. Ipatinga, MG, 2009.

\begin{tabular}{ccc}
\hline $\begin{array}{c}\text { PERCENTIL DO } \\
\text { PERIMETRO CEFÁLICO }\end{array}$ & N & $\%$ \\
\hline$<10$ & 1 & 5,0 \\
$10-90$ & 19 & 95,0 \\
$>90$ & 0 & 0 \\
\hline TOTAL & 20 & 100 \\
\hline
\end{tabular}

A medida do perímetro cefálico $(\mathrm{PC})$ e, consequentemente, o estudo antropométrico do crânio são fundamentais para a avaliação do desenvolvimento de um recém-nascido, pois auxilia na comparação com parâmetros existentes de normalidade ou anormalidade, o que contribui para um acompanhamento mais preciso no caso da detecção de algum problema. Apesar de a medida do PC ser um método simples, sua avaliação é de suma importância, pois seu valor pode fornecer um diagnóstico precoce de anomalias que estão associadas a macrocrania, microcrania, hidrocefalia e atraso ou desaceleração de crescimento. ${ }^{4} \mathrm{~A}$ evolução do perímetro cefálico demonstra o crescimento e a maturação do sistema nervoso. ${ }^{12}$ Dentre as patologias que acarretam alterações na evolução do PC destaca-se a espinha bífida (EB), uma malformação congênita decorrente de um defeito de fechamento do tubo neural que pode ter como complicações os distúrbios neuromotores devidos ao aparecimento da hidrocefalia. ${ }^{17}$ No grupo estudado, não havia nenhum caso de EB.

No Gráfico 2 pode-se observar a distribuição das crianças em relação à classificação do seu desenvolvimento, de acordo com os marcos do desenvolvimento infantil. Segundo a classificação, 1 criança (5,0\%) apresentou "Provável atraso"; 4 (20,0\%), "Possível atraso"; e 15 (75,0\%), "Desenvolvimento normal".

Gráfico 2 - Classificação das crianças de acordo com a avaliação dos marcos do desenvolvimento infantil. Ipatinga,

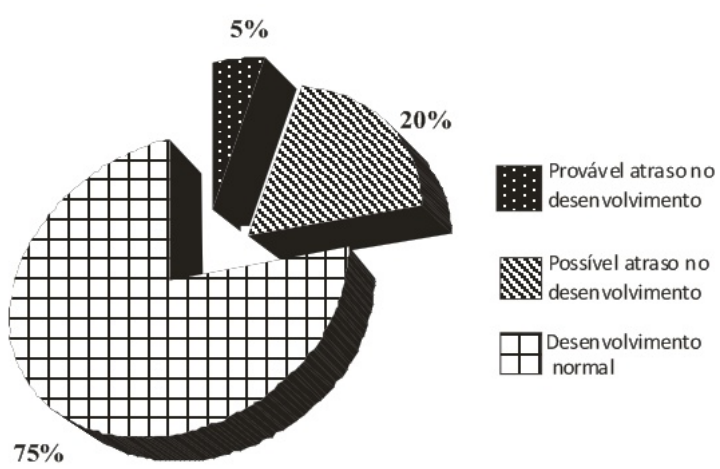


Para as crianças de 2 meses a 2 anos de idade, a ficha de avaliação classifica como provável atraso no desenvolvimento quando o percentil do perímetro cefálico está menor que 10 ou maior que 90, há presença de alterações fenotípicas ou ausência de cumprimento de um ou mais marcos para a faixa etária anterior. ${ }^{1}$ A criança da amostra avaliada e classificada com provável atraso para o desenvolvimento apresentou percentil de perímetro cefálico menor que 10 , além de não conseguir identificar dois objetos, rabiscar espontaneamente, produzir três palavras e andar para trás, marcos que devem estar presentes em sua faixa etária anterior.

A criança será classificada como apresentando um possível atraso no desenvolvimento quando houver ausência de um ou mais marcos para a sua faixa etária ou quando se perceber a existência de um ou mais fatores de risco como baixo peso ao nascer, prematuridade, consanguinidade dos pais, casos de deficiência na família e violência doméstica. ${ }^{1}$ Todos estes fatores foram apresentados pelas quatro crianças classificadas como com possível atraso no desenvolvimento.

\section{CONCLUSÕES}

Percebeu-se com esse estudo que a vigilância do desenvolvimento infantil realizada através da estratégia AIDPI é de suma importância, já que este instrumento fornece resultados eficazes e engloba vários aspectos importantes, como: levantar informações com as mães ou responsáveis sobre fatores de risco para o atraso no desenvolvimento; identificar a percepção da mãe sobre o desenvolvimento de seu filho; verificar as medidas do perímetro cefálico; identificar presença de alterações fenotípicas através do exame físico; e avaliar o desenvolvimento motor grosso, motor fino, linguagem e interação pessoal. Além disso, ele fornece, prontamente, resultados capazes de determinar se a criança está se desenvolvendo como o esperado para sua idade cronológica.

Tal instrumento foi considerado um facilitador da assistência às crianças e da comunicação entre o profissional e a mãe (família), pois utiliza uma linguagem simples e de fácil entendimento. No entanto, foi identificado como ponto negativo para sua aplicação o seguinte aspecto: a ficha de avaliação apresentada pelo manual da AIDPI não traz os marcos do desenvolvimento organizados de forma mensal e, sim, por períodos etários. Sabe-se que as crianças, principalmente as que se encontram na faixa etária de 0 a 2 anos, estão em intenso ritmo de desenvolvimento, o que pode ser percebido mês a mês. Não ter uma ficha que retrate todos esses 24 meses, separadamente, é uma dificuldade adicional na hora de classificar.

Das 20 crianças avaliadas, $75 \%$ foram consideradas como apresentando um desenvolvimento normal, adequado para a idade; $20 \%$ foram classificadas como com possível atraso no desenvolvimento e $5 \%$ com provável atraso no desenvolvimento. Os pais ou responsáveis das crianças que obtiveram resultados com suspeita de atraso foram orientados segundo a estratégia
AIDPI. Além disso, foi encaminhada uma carta a cada responsável pela criança com o resultado da avaliação feita e com prescrições de estimulação do desenvolvimento infantil para serem realizadas mês a mês.

Evidencia-se, portanto, que a avaliação do desenvolvimento preconizado pela estratégia AIDPI deve fazer parte das ações do enfermeiro na consulta de puericultura como um instrumento de triagem para a detecção precoce de atrasos no Dl. Além disso, cabe aos enfermeiros orientar os pais e familiares na busca de promover um ambiente seguro e facilitador para que as crianças alcancem seu potencial de desenvolvimento.

\section{REFERÊNCIAS}

1. Organização Pan-Americana da Saúde - OPAS. Manual para vigilância do desenvolvimento infantil no contexto da AIDPI. Washington, DC; 2005.

2. Leão E, et al. Pediatria ambulatorial. $4^{\mathrm{a} e d}$. Belo Horizonte (MG): Coopmed; 2005.

3. Mussen PH, Conger JJ, Kagan J. Desenvolvimento e personalidade da criança. Tradução de Maria Lúcia G. Leite Rosa. $3^{\mathrm{a}}$ ed. São Paulo (SP): Harbra; 1995.

4. Behrman RE, Kliegman R. Nelson: princípios de pediatria. Tradução de Marcio Moacyr de Vasconcelos. $4^{\mathrm{a}}$ ed. Rio de Janeiro (RJ): Guanabara Koogan; 2004.

5. Instituto Brasileiro de Geografia e Estatística-IBGE. Contagem da população 2007. Rio de Janeiro (RJ); 2007.

6. Ministério da Saúde (BR). Saúde da criança: acompanhamento do crescimento e desenvolvimento infantil. Brasília (DF); 2002. Série Cadernos de Atenção Básica $n^{0} 11$. Normas e Manuais Técnicos.

7. Ministério da Saúde (BR). Capacitação para comitês de ética em pesquisa. Brasília (DF); 2006.

8. Secretaria de Estado de Saúde (MG). Observatório de Saúde de Minas Gerais: microrregião Ipatinga. Belo Horizonte (MG); 2006.

9. Ministério da Saúde (BR). Manual técnico de pré-natal e puerpério: atenção qualificada e humanizada. $3^{\mathrm{a}}$ ed. Brasília (DF); 2006.

10. Fundo das Nações Unidas para a Infância-UNICEF. Situação da infância brasileira 2006 - crianças de até 6 anos: 0 direito à sobrevivência e ao desenvolvimento. Brasília (DF); 2005.

11. Ministério da Saúde (BR). Saúde Brasil 2004: uma análise da situação de saúde. Brasília (DF); 2004.

12. Ramos HAC, Cuman RKN. Fatores de risco para prematuridade: pesquisa documental. Esc Anna Nery Rev Enferm 2009 abr/jun; 13 (2): 297-304. 
13. Knoblock H, Passamanick B. Gessell e Amatruda psicologia do desenvolvimento do lactente e criança pequena: bases neurológicas e comportamentais. São Paulo (SP): Atheneu; 2002.

14. Faria LTM. Abordagem multidisciplinar no acompanhamento de uma criança com traumatismo crânio-encefálico. Análise psicológica. [on-line] 2006 abr; [citado 20 mar 2009]; 24 (2): [aprox: 10 telas]. Disponível em http://www.scielo.br.

15. Wong DL. Whaley \& Wong: enfermagem pediátrica, elementos essenciais à intervenção efetiva. $5^{\mathrm{a}}$ ed. Rio de Janeiro (RJ): Guanabara Koogan; 1999.

16. Motta AFEM, Silva PAG, Araújo CO, Lira IP, Lima CM. 0 peso ao nascer influência o estado nutricional ao final do primeiro ano de vida. J Pediatr. [on-line] 2005; [citado 20 mar 2009]; 81 (5): [aprox. 5 telas]. Disponível em: http://www.scielo.br.

17. Moore KL, Persaud TVN. Embriologia clínica. $6^{a}$ ed. Rio de Janeiro (RJ): Guanabara Koogan; 2000.

18. Gaiva MAM, Neves $A Q$, Siqueira FMG. 0 cuidado da criança com espinha bífida. Esc Anna Nery Rev Enferm 2009 out/dez; 13 (4): 717-25. 